\title{
Sísifo de Marcus Accioly: une poétique de l'épopée moderne \\ ou « de la nouvelleancienne douleur en nouveau style héroïque ${ }^{1}$
}

\section{Rita Olivieri-Godet}

(Université Rennes 2)

Resumo: Le but de cette étude est de mettre en avant les caractéristiques d'une poétique de l'épopée moderne basée sur l'articulation des éléments traditionnels de l'épopée et de ceux de la poétique contemporaine, à partir d'une lecture de Sísifo (1976) du poète brésilien Marcus Accioly (1943-). Auteur de plusieurs ouvrages qui marquent un renouvellement du genre épique, ce poète développe également une réflexion théorique sur son actualité. Cette réflexion nous aidera à penser le parcours de ce genre et la tension entre tradition et innovation qui traverse l'épique moderne.

[...] the historical sense compels a man to write not merely with his own generation in his bones, but with a feeling that the whole of the literature of Europe from Homer and within it the whole of the literature of his own country has a simultaneous existence and composes a simultaneous order.

T. S. Elliot

Marcus Accioly se place au plus haut rang des poètes contemporains de langue portugaise. D'une extraordinaire ampleur et diversité, son œuvre surprend par sa verve étincelante et le merveilleux pouvoir incantatoire qui se dégage de la musique de ses poèmes. Toutes les possibilités de la langue y sont explorées, avec rigueur et émotion, pour faire jaillir une poésie qui s'épanouit dans la plénitude de ses belles images et de son rythme magique, à la recherche d'une expression esthétique susceptible d'atteindre l'apothéose de la langue. Sa poésie naît de l'épiphanie de la langue,

1 Pour le titre, nous avons repris un vers de Latinomérica de Marcus Accioly « da novantiga dor em novo estilo heróico». In Latinomérica, Rio de Janiro, 2001, p. 68. 
l'un des deux versants de la poésie actuelle, l'autre ayant trait à une poétique du silence. ${ }^{2}$ À l'opposé de la concision elliptique, la diction paroxystique de Marcus Accioly est révélatrice de sa façon de vivre l'expérience poétique en tant que jouissance de la parole. Chez Accioly, poète, critique et avant tout, lecteur anthropophage, l'épiphanie de la langue s'accomplit dans la plus stricte conscience critique du processus de création, maîtrise technique et génie de l'invention se combinant pour extraire la poésie de la langue. Cette complémentarité, assez rare chez les poètes contemporains, fait de lui un des poètes les plus complets de son époque.

Inscrite au carrefour d'un héritage classique et populaire, l'œuvre de ce poète et théoricien de la poésie puise ses modalités de création dans la tradition littéraire occidentale, célébrée et reconnue, de même que dans la poétique populaire du Nordeste du Brésil qui maintient encore vivante la tradition de la composition orale. Son génie poétique s'approprie cette mémoire littéraire d'une façon tout à fait novatrice, en se frayant le chemin du dialogue. Ainsi, il se distancie de ceux qui se réfugient dans l'attitude stérile du culte de la tradition comme de ceux qui refusent de la prendre en compte. Dans le sillage de T. S Elliot, l'auteur refuse de considérer la tradition comme un héritage passif, l'écrivain ne pouvant y accéder qu'à travers une connaissance conditionnée par l'usage novateur qu'il est capable d'en faire. Par conséquent, sa place dans la contemporanéité ne peut être perçue que dans une perspective historique.

La poésie de Marcus Accioly ainsi que ses réflexions théoriques, comme le témoignent certains passages de son livre qui fait figure de manifeste intitulé Poética. Pré-manifesto ou anteprojeto do realismo épico (1977), révèlent un poète imbu du «historical sense» ${ }^{3}$ auquel se réfère Eliot. Partant, au lieu de se borner aux seules circonstances de son temps et de sa génération, Accioly - poète dépositaire de la mémoire poétique universelle et brésilienne -

2 Voir à ce sujet, le poète et théoricien portugais Alberto Pimenta, O silêncio dos poetas, Lisboa: Cotovia, 2003, p. 70-72.

3 Sur ce sujet, je renvoie au célèbre essai de T.S Eliot, "Tradition and individual talent ", The sacred wood, Essays on poetry and criticism, Methuen: London; Barnes $\S$ Noble: New York, 1960, p. 47-59. 
envisage le temps dans sa totalité: «le temps - comme la corde d'un instrument de musique - doit être joué dans sa totalité et ce qui est important c'est le tempo avec lequel nous touchons son fil» («o tempo - como a corda de um instrumento musical - deve ser tocado por inteiro e o que importa é o «tempo» em que tocamos seu fio») ${ }^{4}$; «le poète doit être entier pour être lui-même et, être entier signifie qu'il est un - tout.» («o poeta tem que ser ele todo, para que seja ele mesmo e, ser ele todo significa ser ele um - todo.. ${ }^{5}$

Ce désir de s'installer dans la totalité du temps (but impossible à atteindre mais toujours poursuivi) le conduit tout naturellement à celui de pouvoir tout exprimer, d'où la préférence pour les longs poèmes, la nature fortement intertextuelle de son discours, la multiplication des rôles assumés par le moi, la richesse et la diversité des procédés poétiques que l'on retrouve dans son oeuvre. Le poète s'installe dans la totalité de la vie du langage, dans un effort gigantesque pour embrasser la diversité de l'expérience humaine: il construit ses ouvres comme des sommes, comme s'il voulait rassembler toutes les nuances de la problématique existentielle de l'homme, tous ses sentiments, ses espoirs et ses erreurs. Ce désir de totalité peut-être expliqué par la présence de l'archétype de l'unité primordiale qui hante la poésie moderne. ${ }^{6}$ Il s'exprime par l'évocation fragmentaire de différents champs de l'imagination, de différentes nuances de l'affectivité humaine. Pour le poète moderne, la totalité ne peut se traduire que par la pluralité et la fragmentation.

Il est significatif que le poète choisisse de bâtir sa trilogie épique autour de trois personnages en proie à d'affreux tourments Sisyphe, Ixion et Narcisse. Ce choix de trois personnages «maudits et mythologiques», selon les propres mots de l'auteur, révèle une vision particulière de la condition humaine, dans laquelle l'homme est habité par le désir d'échapper à ses souffrances et de dépasser son intense insatisfaction vis-à-vis de sa situation actuelle. La trilogie épique

4 Marcus Accioly, Poética. Pré-manifesto ou anteprojeto do realismo épico. Recife: Editora Universitária, 1977, p. 14.

5 Ibid. p. 14.

6 Voir à ce sujet, l'ouvrage de Roberval Pereyr (2000). A unidade primordial da lírica moderna, Feira de Santana: UEFS, 2000. 
articule par ailleurs les dimensions mythiques et historiques du réel. Il ne s'agit pas d'un pur mythologisme, d'un simple procédé de transposition limité à la lecture du monde que proposaient les mythologies antiques. Cette trilogie est investie d'une fonction d'historicisation du mythe, qui met en relief la façon dont le mythe nous parle aujourd'hui, la manière dont ses débris persistent et touchent l'homme de notre temps.

Sísifo (1976) est le premier ouvrage de la trilogie mythologique de Marcus Accioly. Suivi d'Íxion (1978) et de Narciso (1984), il inaugure une poétique du réalisme-épique, selon la terminologie de l'auteur lui-même qui, jouant sur les mots (épique époque), souligne par ce rapprochement son choix d'un discours épique imprégné d'une conception du temps qui rattache l'auteur à son époque, sans que pour autant les liens avec le passé soient coupés. Pour mieux comprendre le dialogue singulier que l'auteur entretient avec la tradition, il faut se pencher sur cette cuvre pour dégager les éléments qui témoignent d'une conception littéraire moderne de l'épique.

L'engouement de la critique pour Sísifo peut être observé au travers des propos qui voient dans cette œuvre en langue portugaise une épopée de notre époque capable d'intégrer, dans une nouvelle parole poétique, l'héritage de la culture occidentale (Nelly Novaes Coelho), ou un modèle de l'épique moderne (Silva, 1994) ou bien encore la plus grande réalisation de la génération 60 (LYRA, 1995).

Poème en dix chants, la structure générale de Sísifo présente les parties fondamentales de l'épopée classique: ouverture, proposition, invocation, dédicace et narration. Mais, au-delà des ressemblances explicites et des innovations palpables comme celle de la double invocation païenne et chrétienne qui annonce le point de vue élargi de la voix poétique désireuse d'englober les traditions distinctes qui forment l'imagination des hommes et leurs systèmes symboliques, il faut examiner ce qui constitue pour Accioly la matière épique et la manière dont il la façonne pour créer son propre univers littéraire.

Dans cette épopée, les éléments structurels et sćmantiques s'organisent autour de la figure mythique de Sisyphe. Le supplice de Sisyphe, condamné à rouler éternellement un rocher vers le sommet d'une pente, constitue le noyau poétique de l'œuvre, point de 
convergence d'une parole exubérante, ambitieuse dans son désir de revisiter les mythes mais aussi l'histoire, la tradition littéraire, philosophique et scientifique de la culture occidentale. Il s'agit bien d'un noyau, mais d'un noyau éclaté; il n'est pas question de reprendre un déroulement linéaire de l'histoire du mythe, il ne s'agit pas de raconter sa légende, même si le lecteur finit par la reconstituer à partir des éléments fragmentaires fournis par le poème, mais de réinterpréter le mythe en explorant ses différentes composantes, en les dotant d'une nouvelle force symbolique, en les transformant, voire en en inventant d'autres.

La vision du châtiment de Sisyphe hante la littérature depuis son apparition première dans l'Odyssée d'Homère. Marcus Accioly en fait le leitmotiv de la construction de son texte épique tout en déplaçant le sens traditionnel du mythe. Sisyphe n'est plus envisagé dans son cercle tragique, victime d'un mouvement vain, toujours égal à lui-même; il est plutôt l'homme en continuel devenir. L'auteur y intègre la lecture qu'en fait Albert Camus pour qui «la lutte elle-même vers les sommets suffit à remplir un cœur d'homme. Il faut imaginer Sisyphe heureux»). Pour Camus (1942, p. 166), l'éveil de la conscience sur la condition humaine permet à l'homme de donner du sens à l'absurde de la vie, d'accepter le châtiment en imposant un sens à l'effort vain. Accioly s'inscrit en faveur de cette perspective de Camus lorsque, dans son actualisation du mythe, il projette l'expérience poétique du langage comme un désir de dépassement de l'effort vain.

ah eu te peço neto por Antíope

(e por mim) não me penses a loucura

de levar esta pedra na montanha

pois loucura maior era não tê-la

e muitos não a têm Foco e não sabem

que é bastante uma pedra para um homem

levar durante a vida e muitos levam

a vida toda tendo as mãos vazias ${ }^{7}$

Le nouveau Sisyphe (poète) forme un tout avec son rocher (poème), il ne peut pas s'en passer, son châtiment est aussi sa délivrance. Ainsi, la matière épique du Sísifo de Marcus Accioly se 
construit autour de l'expérience poétique du langage. Il y est question de l'acte créateur qui imprime du sens à la vie, geste éternel que l'artiste ne cesse d'entreprendre dans son effort de recréer le langage pour découvrir et refaire le monde. Dans sa dédicace, la voix poétique explicite son dessein de chanter l'acte créateur, en rendant hommage à la mémoire de tous ceux qui ont écrit «un chant intemporel» et qui l'ont précédé dans l'invention de Sisyphe. Ce faisant, elle situe le poète dans un entre-lieu entre continuité et rupture, souligné par le fait qu'au tout début de la «narration», ${ }^{8}$ il attribue à la lecture la fonction de source première de la poésie. Création et lecture ne sont en fait que les deux facettes d'un même processus, celui du décryptage du monde par l'expérience poétique du langage.

Ainsi pourrait-on dire que le récit épique de ce poème se fait autour de la relecture de ses sources et des interrogations que sa propre facture engendre sur les rapports entre l'homme, le langage et le monde. Épopée sans intrigue, ce poème est conçu comme une mise en abyme de l'acte créateur ainsi que le révèlent les titres attribués aux dix chants qui le composent: A Irrealidade Mitológica; O Possivel da Lenda; $O$ Impossível da Alegoria; A Revelação Irrevelada; Polissísifo; A Consciência Inconsciente; A Paz-Guerra; A Irreal-Irrealidade; O Oficio do Oficio; A Reinvenção do Mito. À travers tout un monde créé par le langage, c'est la fable du réel que Marcus Accioly compose.

Cette conscience du processus de création, qui se manifeste tout au long du poème, est prolongée dans les notes que l'auteur adresse au lecteur, ce qui confirme l'intention de ce poète-théoricien de rapprocher l'acte créateur d'une réflexion critique. Les notes éclairent des éléments de la genèse du texte, dévoilent des références bibliographiques, suggèrent des schémas de lecture en jouant sur l'ordre des mots, expliquent et interprètent certains passages du texte poétique. De cette manière, elles multiplient les indices sur le travail accompli par l'auteur dans le texte et permettent de mieux le situer par rapport aux sources diverses de la culture occidentale qui le nourrissent.

8 En réalité, il s'agit plutôt de débris de narration qui ne correspondent ni à la forme, ni à la fonction de cette partie dans l'épopée classique. 
Nous sommes donc face à un poème de nature profondément méta-poétique qui expose son désir de revisiter la tradition poétique universelle et brésilienne, depuis les textes fondateurs jusqu'aux expérimentations avant-gardistes (Canto 5, Polissísifo), en explorant tous les recours de l'intertextualité (citations, paraphrases, pastiches, parodies, allusions) pour réinventer un discours nouveau. Dès l'ouverture du poème, le poète étale son intention de dialoguer avec la tradition littéraire par le croisement des citations des vers de Dante et de Camões et des allusions aux chants d'Homère et de Virgile:

Da nossa vida em meio da jornada

entre armas e barões assinalados

tendo perdido a verdadeira estrada

vi-me por mares nunca navegados

selva selvagem Tróia incendiada

muitos heróis nas guerras esforçados:

Aquiles Odisseus Vascos Enéas

ó comédias tragédias epopéias ${ }^{9}$

La voix poétique manifeste l'aspiration à se fondre dans cette mer de paroles pour s'en départir aussitôt et inscrire sa propre diction dans la tradition littéraire. L'image du poète affamé de mots s'impose aux yeux du lecteur qui s'apprête lui-aussi à refaire la découverte du monde par la poésie, guidé par ce geste qui ne cesse d'inaugurer "l'impossible dans le possible». ${ }^{10}$ Loin de la distanciation caractéristique du poète-narrateur épique classique, qui garde un certain recul par rapport à sa matière, le lecteur est confronté à une voix qui inaugure une expression subjective du monde et se situe dans une a-temporalité caractéristique du lyrisme:

Piso da terra para o espaço pleno

meus pés vacilam entre os dois abismos

porém firmando as mãos o céu ordeno:

é preciso subir quando é preciso

sei que a montanha é alta e eu sou pequeno

9 Sísifo, Canto Primeiro, versos 1-8.

10 Sísifo, Canto Segundo, versos 79-80 "[...] (no alegórico / o impossível é dentro do possivel)". 
ó descida ó estágio ó alpinismo rolarei para sempre (novo Sísifo)

a pedra do poema até os píncaros ${ }^{11}$

Le poème actualise le mythe de Sisyphe et le thème traditionnel de la descente aux enfers. Comme Sisyphe, la voix poétique fait l'expérience du gouffre, de la «saison en enfer» pour pouvoir accéder aux cimes par l'acte créateur qui le libère. L'analogie qui se développe tout au long du poème et qui identifie le poète à un nouveau Sisyphe s'appuie sur des images qui évoquent ce mouvement d'ascension et de descente. Ainsi, le poème articule les pôles contradictoires et complémentaires de l'expérience humaine: souffrance et libération, passé et présent, enfance et maturité de l'homme dans un mouvement cyclique comme celui du cosmos, toujours recommencé:

mundo dentro do mundo (universo de Sísifo
cujo fim da Galáxia é o próprio princípio)

Sisyphe-poète est le héros du poème épique de Marcus Accioly qui élabore, par ce choix allégorique, un chant au pouvoir fondateur du langage: "Sisyphe est un Dieu sur la montagne», écrit l'auteur ${ }^{13}$. Le je, présent dans le texte, structure le discours et assume toutes sortes de masques, instaurant de cette manière une parole errante qui se manifeste et se transforme pour dépeindre les multiples et paradoxales facettes de l'être humain. Ce je n'a pas d'identité fixe, il se projette dans son poème, il n'existe qu'à travers lui. Poète et poésie ne font qu'un, ils se renvoient leurs images et confondent leurs voix, comme dans ce passage qui évoque le rêve du rocher:

não sou tão só

em mim há tanta gente

11 Ibid., Canto Primeiro, versos 25-32.

12 Sísifo, Canto Primeiro, vers 620-621.

13 «Sísifo é um deus na montanha, Íxion é um demônio à roda, Narciso é simplesmente um homem na água, um feto, um narciso e - mais que o Estêvão do conto de Gabriel García Márquez - ele é o afogado mais bonito do mundo." Voir Marcus Accioly, "Notas ao leitor», Narciso, Recife: Fundação de Cultura da Cidade de Recife / Rio de Janeiro: Francisco Alves, 1984, p. 300. 
tanto dos outros tanta dor alheia
tanta pena que eu sinto por quem sente
tanta mágoa encontrada pela vida
tanto amor que eu somei e dividi
e tanta angústia que não era minha
e tanta solidão que eu já não tinha
tantos destinos que eu não sou mais eu ${ }^{14}$

Symbole ou voix, le rocher s'interroge sur son identité, aussi flottante, inconstante et polyphonique que celle du poète.

L'errance identitaire de la voix poétique est inscrite dans la matérialité même du poème. Elle va de pair avec la diversité formelle du texte. La fragmentation sémantique, qui renonce à l'intrigue au profit d'un déploiement d'images poétiques, s'appuyant souvent sur les procédés d'association d'images et d'énumération, est suivie par ce que l'auteur appelle «la fragmentation des formes adoptées». D'après lui, celle-ci renvoie à «la fragmentation du monde moderne qui, pétale par pétale, désintègre la fleur du chaos». ${ }^{15}$ C'est ainsi que la voix poétique se dédouble dans différents tons, même si le ton solennel prédomine dans l'ensemble du poème. Elle se laisse apprivoiser par toutes sortes de rythmes: si l'alexandrin et le décasyllabe constituent une sorte d'arrière plan rythmique (dans certains cas, les césures, doublées de la disposition graphique des vers, ajoutent une touche moderne à la tradition), le système général du poème s'appuie sur une polymétrie à laquelle vient s'additionner une variété strophique. Cette variété de strophes et de vers explore librement l'espace graphique de la page pour produire du sens, dans le sillage des avant-gardes poétiques européennes et brésiliennes. Ainsi, cette voix affamée de mots ne peut être que plurielle. Elle fonde une poétique du fragmentaire qui déplace les éléments consubstantiels à l'épique traditionnel: l'unité de la narration d'un événement qui structure l'action, la situe dans un espace précis et donne vie aux personnages.

Poème-énigme, Sísifo est Edipe face au sphinx; poèmemiroir, Sísifo est Narcisse obsédé par sa propre image:

14 Sísifo, Canto Terceiro, "a pedra sonha", vers 136-145.

Sísifo, «Notas para o leitor leitor», p. 391. 


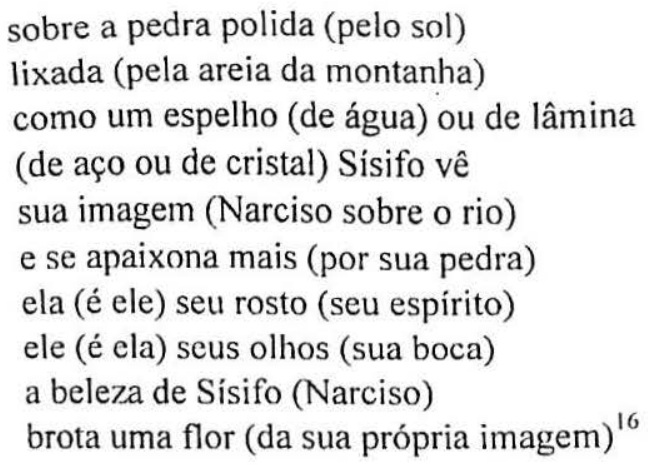

Marcus Accioly choisit un genre qui peut paraître anachronique à une époque (années 70) où la poésie brésilienne se complaît dans l'expérimentation du langage ou dans la recherche d'une expression anti-lyrique ou encore dans une poétique du silence. La sensibilité historique de son épopée-palimpseste incorpore tous ces versants contemporains à sa propre production. Mais, cet aède moderne a assez d'étoffe pour construire un poème de cette envergure où l'effort intellectuel requis par un projet qui revisite la tradition culturelle occidentale est soumis au filtre d'une expression subjective lyrique. Érudition et émotion, sensibilité historique et affective se conjuguent pour imprimer à la matière épique primitive un détour novateur qui surprend et enchante le lecteur: ce chant qui jaillit d'une «nouvelleancienne douleur en nouveau style hérö̈que» est une invitation à faire de l'expérience poétique un moyen privilégié pour regarder "la montagne mythique du monde» et pénétrer dans ses mystères abyssaux. Épopée du langage, Sisifo est source de vie. 


\section{Références}

ACCIOLY, Marcus. Sísifo. São Paulo: Quíron; Brasília: INL, 1976. ACCIOLY, Marcus. Íxion. Rio de Janeiro: Tempo Brasileiro, 1978. ACCIOLY, Marcus. Narciso. Recife: Fundação de Cultura da Cidade de Recife; Rio de Janeiro: Francisco Alves, 1984, ACCIOLY, Marcus. Latinomérica. Rio de Janeiro: Topbooks, 2001. ACCIOLY, Marcus. Poética. Pré-manifesto ou anteprojeto do realismo épico. Recife: Editora Universitária, 1977.

BRUNEL, Pierre; BASTIAN, Aeneas. Sisyphe et son rocher. Paris: Éditions du Rocher, 2004.

CAMUS, Albert. Le mythe de Sisyphe. Paris: Gallimard, 1942. (Idées) ELIOT, T.S. Tradition and individual talent, The sacred wood, Essays on poetry and criticism. Methuen: London; Barnes § Noble: New York, 1960

ELIOT, T.S. A essência da poesia. Rio de Janeiro: Artenova, 1972.

LYRA, Pedro. Sincretismo: a poesia da geração 60. Rio de Janeiro: Topbooks, 1995.

MORICONI, Ítalo. A poesia brasileira do século XX. Rio de Janeiro: Objetiva, 2002.

PEREYR, Roberval. A unidade primordial da lírica moderna. Feira de Santana: UEFS, 2000.

PIMENTA, Alberto. O silencio dos poetas. Lisboa: Cotovia, 2003

SILVA, Anazildo Vasconcelos. Semiotização literária do discurs. Rio de Janeiro: Elo Editora, 1984. 